\title{
A numerical study of multi-pass design based on Bezier curve in conventional spinning of spherical components
}

\author{
Tian Gan ${ }^{1}$, Qingshuai Kong ${ }^{1}$, Zhongqi Yu ${ }^{1}$, Yixi Zhao ${ }^{1}$ and Xinmin Lai ${ }^{1}$ \\ ${ }^{1}$ Shanghai Jiao Tong University, China
}

\begin{abstract}
Bezier curve has been widely used as the roller path in conventional spinning. In this paper, Finite Element model with parameterized 2-pass conventional spinning tool paths based on quadratic Bezier curve is developed to explore the influence on the wall thickness reduction of spherical components. The results show that the first path has significant effect on wall thickness distribution and the selection of parameter values in tool path is crucial for thickness distribution.
\end{abstract}

\section{Introduction}

Conventional spinning is defined as the process with a constant thickness but a changeable diameter. Its inherent advantages, such as high quality surface finish, low forming forces and process flexibility [1], make it widely used in automotive and aerospace industries. Single onepass conventional spinning, limited by forming limits, tends to wrinkles formation and fracture occurrence. In the view of Runge [2] and Lange [3] works, multi-pass should be performed in conventional spinning in order to form the blank without defects. In multi-pass conventional spinning, the shape of tool path in each pass plays an important role in achieving the targeted geometries.

Many studies have been carried out on roller tool path design. Various tool paths such as linear, concave and convex have been analysed. Hayama et al. [4-6] concluded that concave tool paths give the best results and involute paths were recommended. Kang et al. [7] experimentally analysed the above three types of tool paths and stated that the deformation in the first pass has a decisive effect on the wall thickness distribution of the product when the original blank is a plate. Wang and Long [8] numerically analysed the effects of four different roller paths on tool forces, wall thickness and stress distribution in conventional spinning. They have concluded that concave roller path resulted in higher reduction of wall thickness. Li et al. [9] developed parameterized roller paths with three times Bezier curve during the first pass of die-less spinning, and observed that with the increase of curvature, the location of severe thinning area moves backwards from the middle part of the blank to the end part. James et al. [10] performed a set of experiments to investigate how tool force and part geometry evolved with the change of parameters of quadratic Bezier curve during the first pass.

\footnotetext{
a Corresponding author: gantian426833@sjtu.edu.cn
}

The published studies give an insight into tool path design in conventional spinning. However, in the previous works, roller paths design were almost focused on one-pass conventional spinning, and limited investigations were carried out on multi-pass processes. Previous works have already demonstrated that concave tool paths gave better results. In this study, Bezier curve is simply applied during the deformation of the flange. Therefore, thickness distributions of spherical components are investigated based on two-pass conventional spinning simulations with concave quadratic Bezier curve in forward pass.

\section{Methodology}

\subsection{Roller paths parameterization}

Control points of the roller paths are defined at the center of roller nose. The tool path is parameterized referring to the study by James et al. [10]. As Figure 2 shows, each forward pass consists of two parts: one is circular arc, which keeps constant clearance between mandrel and roller, the other is quadratic Bezier curve. The definition of the quadratic Bezier curve is:

$$
P(t)=\sum_{i=1}^{2} P_{i} B_{i, 2}(t)=(1-t)^{2} P_{0}+2 t(1-t) P_{1}+t^{2} P_{2} t \in[0,1]
$$

where $P_{i}$ are control points. To facilitate the generation of Bezier curve in Matlab, the matrix form of Bezier curve is used:

$$
P(t)=\left[\begin{array}{lll}
t^{2} & t & 1
\end{array}\right]\left[\begin{array}{ccc}
1 & -2 & 1 \\
-2 & 2 & 0 \\
1 & 0 & 0
\end{array}\right]\left[\begin{array}{c}
P_{0} \\
P_{1} \\
P_{2}
\end{array}\right] \quad t \in[0,1]
$$


The quadratic Bezier curve (Figure 1) is determined by three control points, which constitute the characteristic polygon.

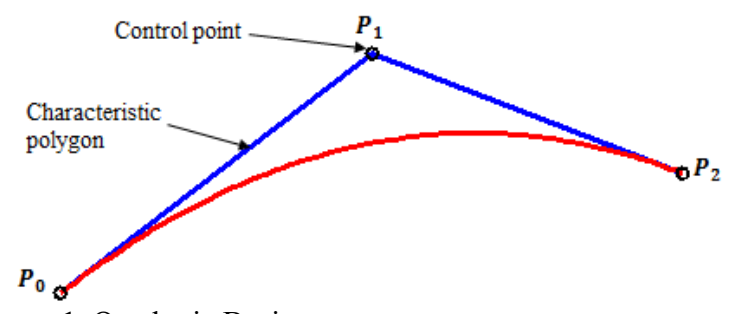

Figure 1. Quadratic Bezier curve.

The Bezier curves used in this study are parameterized in Figure 2. The starting point $P_{0}$ is defined by the end of the constant clearance part of the previous pass and the angle $\theta_{0}$, which is the deformation of adjacent paths. The ending point $P_{2}$ is determined by the axial coordinate $Z_{2}$ measured from the ending point of the previous pass to the end of this pass. Control point $P_{1}$ is defined relative to the mid-point of line $P_{0} P_{2}$, the mid-point moves with $U_{d}$ in the direction of $\mathbf{P}_{\mathbf{0}} \mathbf{P}_{\mathbf{2}}$, then moves with $U_{n}$ in the direction perpendicular to $\mathbf{P}_{\mathbf{0}} \mathbf{P}_{\mathbf{2}}$. The tool path between path 1 and path 2 consists of two straight lines and an arc. The roller does not contact with the workpiece during this process, therefore the control points can be set at any appropriate places.

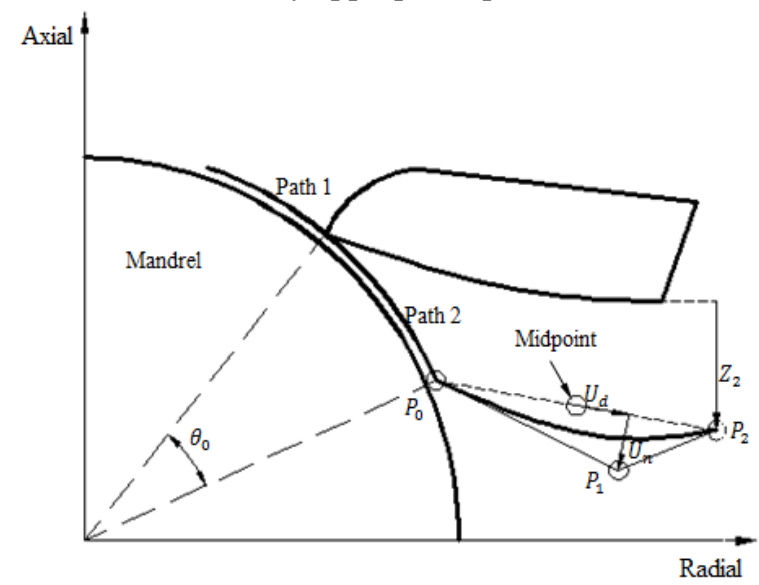

Figure 2. Toopath parameterization.

\subsection{Setting of basic parameters}

The material used in this study is 2024 aluminium alloy, the dimension of the blank is a diameter of $200 \mathrm{~mm}$ with constant thickness of $1.8 \mathrm{~mm}$. A spinning speed of $200 \mathrm{r} / \mathrm{min}$ and a feed rate of $200 \mathrm{~mm} / \mathrm{min}$ are used in both experiment and simulation. The angle between the axis of the mandrel and the axis of the roller is 40 degree. The experiments are all conducted on the horizontal spinning machine. A schematic diagram of spinning experiments is shown in Figure 3.

\subsection{Finite element analysis}

Finite Element models are developed by using commercial FE software Abaqus/Explicit. Due to the large strains and complex contact conditions during this process, the dynamic explicit solver is chosen to analyse the forming process. The roller, back plate and mandrel are modelled as 3D analytical rigid bodies. The blank, the only deformable component, is meshed with 8-node 3D reduced integration continuum shell element. The material properties of the blank are calculated by the mass density as $2780 \mathrm{~kg} / \mathrm{m}^{3}$, elastic modulus as 71000 $\mathrm{MPa}$, and the Poisson's ratio as 0.33. A friction coefficient of 0.5 is set up between the blank and the back plate, 0.02 between the blank and the roller, and 0.2 between the blank and the mandrel. To assure the blank is clamped between the mandrel and the back plate a compressive force of $60 \mathrm{KN}$ is set above the back plate. A mass scaling factor of 25 is used in all models to speed up the Finite Element analysis. The Finite Element model is shown in Figure 4.

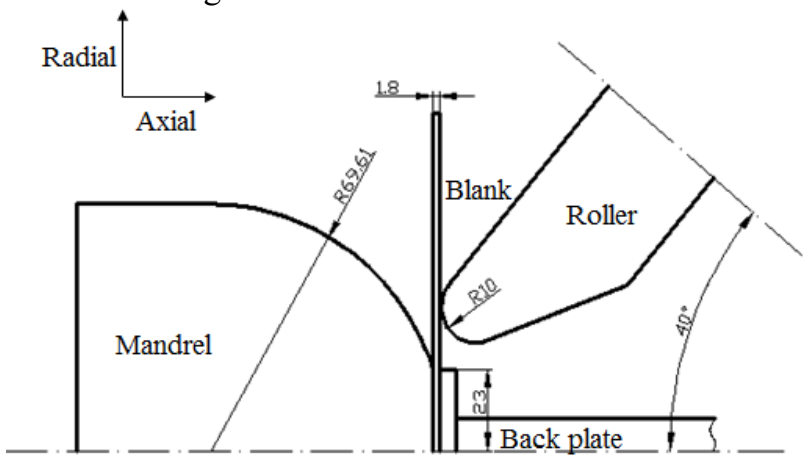

Figure 3. Schematic diagram of the spinning experiment (unit: $\mathrm{mm})$
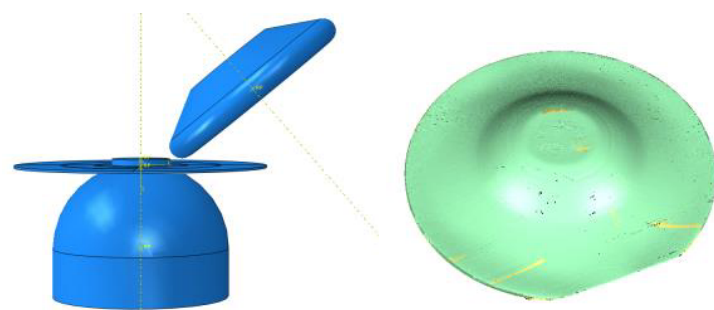

Figure 4. Finite Element model. Figure 5. Data points of experiment

\section{Results and discussion}

\subsection{Verification of the Finite Element model}

The simulation models are verified by comparing with experimental data. A conventional spinning experiment with single pass to 45 degree is applied. A laser scanning $3 \mathrm{D}$ measurement system FARO is used to obtain the coordinates of points at the inner and outer surface of the experiment component, then with the aid of the software Geomagic Qualify the wall thickness distribution is analysed. The data points scanned by FARO are shown in Figure 5. The wall thickness distributions of three Finite Element models with different grid densities of the blank are studied as shown in Figures 6a-6c. The grid densities of model (a), (b) and (c) are 1936, 4956 and 9000 elements. At the same time, the tool paths used in Finite Element models are consistent with the experiments. Figure 6 shows the wall thickness distribution of FE analysis. 


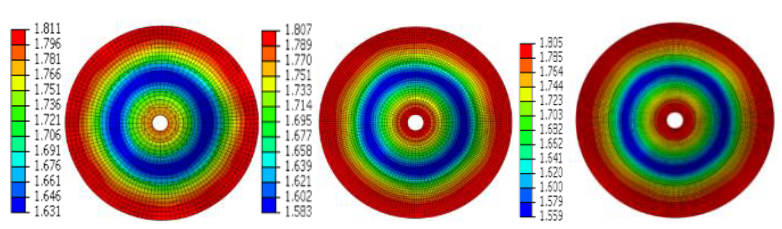

$\begin{array}{lll}\text { (a) } 1936 \text { elements } & \text { (b) } 4956 \text { elements } & \text { (c) } 9000 \text { elements }\end{array}$

Figure 6. Wall thickness distribution of FE analysis.

The wall thickness reductions obtained from the FE analysis models are compared with the result of the experimental sample, as shown in Figure 7. The deviation of the former region is smaller than that of the latter region, and as the grid density increases, the deviation becomes smaller. In order to keep the experiment component fixed during the measurment process, the bottom of the component need to be glued to the holder, thus the wall thickness deviation increases obviously during the radial distance of $0 \mathrm{~mm}$ to $20 \mathrm{~mm}$. The maximum error of the wall thickness reduction between the experimental and simulated values is about $4 \%$. Deviations within $5 \%$ are considered acceptable. Table 1 compares the maximum wall thickness reduction of the experimental part with the simulation results. The result of model (c) shows a good agreement with experimental data.

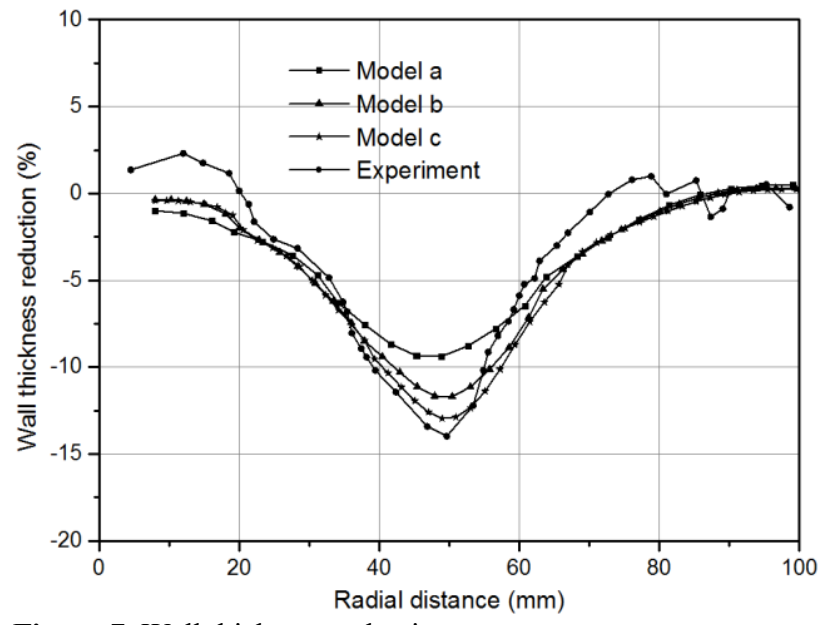

Figure 7. Wall thickness reduction

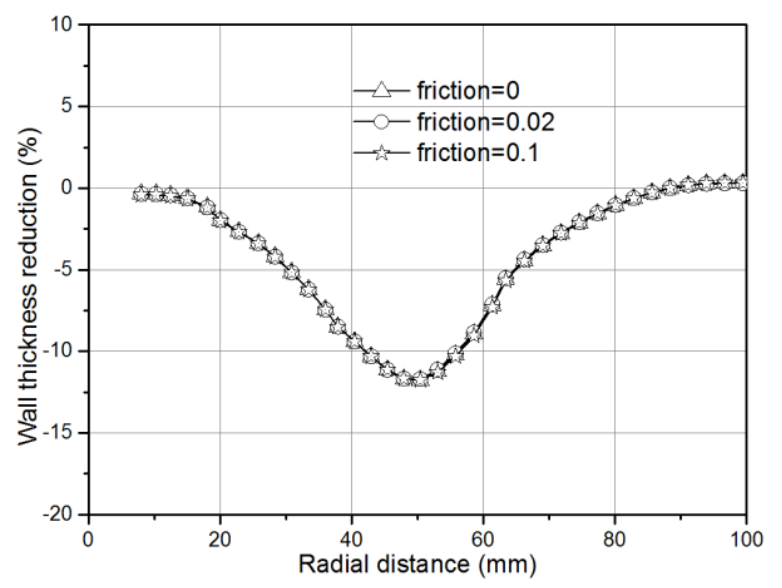

Figure 8. Sensitivity of the results to the friction

In the works of Xia [12], which have investigated the forming forces of tubes spinning using the material of 6061 aluminium alloy, the friction coefficient between the roller and the workpiece is set as 0.1 . The sensitivity of the wall thickness reduction to the friction between the roller and the workpiece is necessary to be analysed. Three FE models with different friction coefficients of 0 , 0.02 and 0.1 are established. As revealed in Figure 8, wall thickness reduction curves with various friction coefficients are almost completely coincide. The friction coefficients of 0.02 used in this study is appropriate.

Table 1. Comparison of FE and experimental results

\begin{tabular}{ccccc}
\hline & Model a & Model b & Model b & Experiment \\
\hline $\begin{array}{c}\text { Max } \\
\text { thinning }\end{array}$ & $9.37 \%$ & $11.69 \%$ & $12.97 \%$ & $13.97 \%$ \\
\hline
\end{tabular}
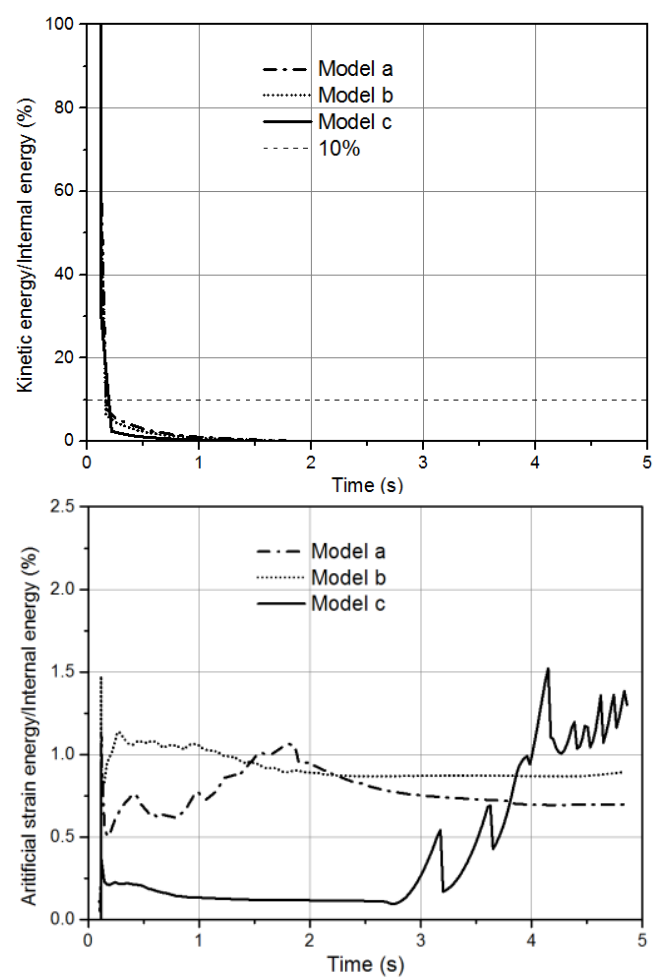

Figure 9. Evaluation of energy histories

The energy histories of the FE models are studied to evaluate the simulation results. To ensure that the simulation results are not significantly affected by mass scaling, the ratio of kinetic energy to the internal energy of the blank should not be greater than $10 \%$ during most time of the process. Moreover, the artificial strain energy should also be less than $5 \%$ of the internal energy to avoid the hourglass problem, which causes the shear locking phenomenon in the reduced integration linear elements and reduces simulation accuracy. As shown in Figure 9, at the beginning of the process, because of the rotational acceleration of the blank at the beginning of the process, the ratio of the kinematic energy to internal energy is much larger than $10 \%$ at that time. However, during most of the process this ratio is below $10 \%$. The ratio of the artificial strain energy to the internal energy is below 5\% throughout the process. Therefore, the dynamic problem caused by mass scaling and the hourglass problem are well controlled. Considering the accuracy of the simulation, model (c) is used for the following Finite Element analysis. 


\subsection{Analysis of the first pass}

The deformation in the first pass has a significant effect on the wall thickness distribution of the product when the original blank is a plate [7], meanwhile a wide ringshaped contact region between blank and mandrel can avoid failures such as wrinkling or fracture. Experiments carried out by Quigley and Monaghan [11] indicated that there was some degree of shear forming involved in the first roller pass of conventional spinning. Therefore, it is certain that clearance exists between mandrel and the inner face of the component. In order to reduce the wall thickness variation of the first pass, three different shapes of the first pass with the original thickness of $1.8 \mathrm{~mm}$ are analysed: (i) conventional spinning to 35 degree with the clearance of $1.8 \mathrm{~mm}$; (ii) conventional spinning to 35 degree with the clearance of $1.65 \mathrm{~mm}$; (iii) shear spinning to 35 degree. Figure 10 presents the thickness reduction of the models, and Figure 11 shows the comparison of clearance. Table 2 is the maximum thickness reduction of the three different first passes. Concluding from the figures that conventional spinning with the clearance of $1.65 \mathrm{~mm}$ has the smallest clearance and the thickness reduction is less than shear spinning. The conventional spinning to 35 degree with the clearance of $1.65 \mathrm{~mm}$ is selected as the first pass.

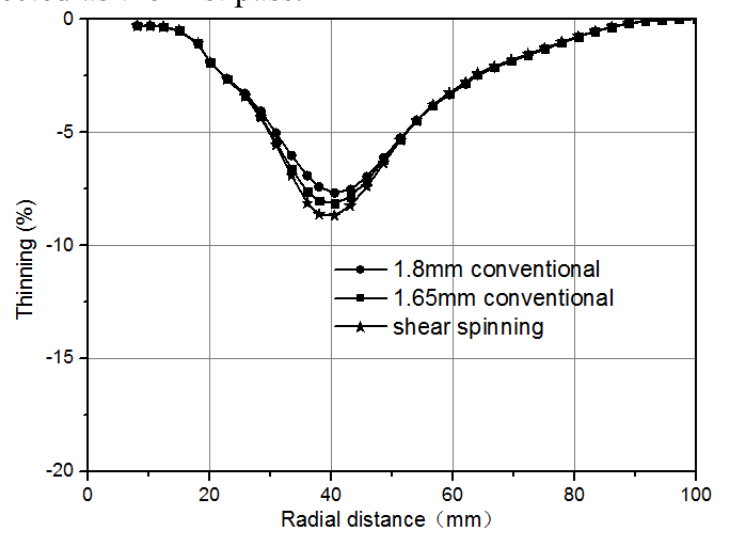

Figure 10. Wall thickness reduction of first pass

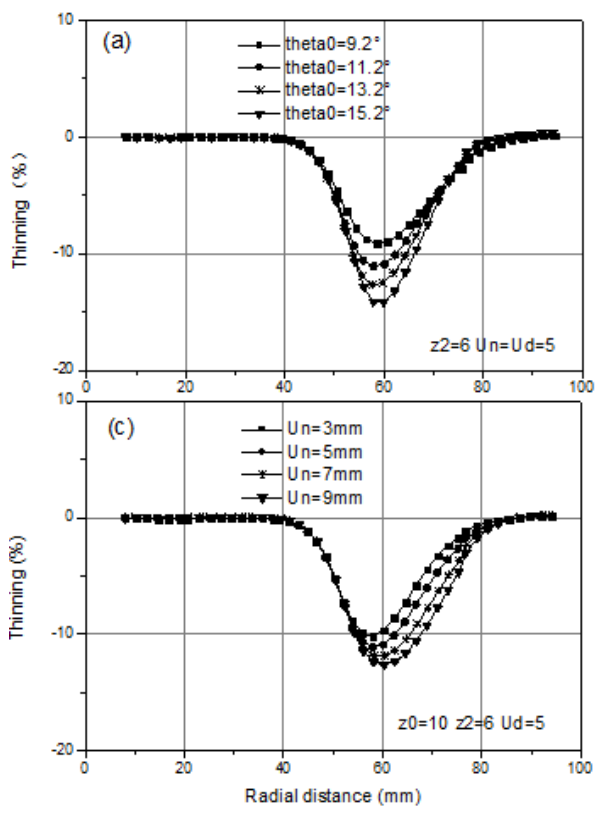

Figure 12. Thickness variation of toolpaths
Table 2. Maximum thinning comparison

\begin{tabular}{cccc}
\hline & $\begin{array}{c}1.8 \\
\text { conventional }\end{array}$ & $\begin{array}{c}1.65 \\
\text { conventional }\end{array}$ & $\begin{array}{c}\text { shear } \\
\text { spinning }\end{array}$ \\
\hline $\begin{array}{c}\text { Max } \\
\text { thinning }\end{array}$ & $7.72 \%$ & $8.20 \%$ & $8.72 \%$ \\
\hline
\end{tabular}
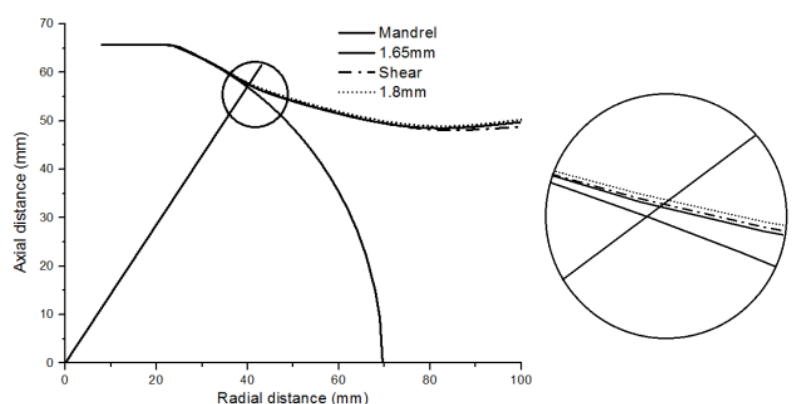

Figure 11. Clearance comparison of first pass

\subsection{Analysis of the second pass}

The thickness reduction varies with different parameters. Figure 12 shows the thinning along the blank of various passes. The result demonstrates that for the second pass, increasing parameters $\left(\theta_{0}, Z_{2}, U_{n}\right)$ leads to increased thinning, particularly in the middle part of the blank. Li Y [9] and James[10] gave the similar conclusion about the thinning phenomenon in die-less spinning. Parameter $\theta_{0}$ is the deformation of adjacent paths, Figure 13 (a) shows the different roller paths with the change of $\theta_{0}$. The radial distance of starting points of Bezier curve, as shown in Figure 13 (a), is between 55 and 60. Figure 13 (a) and Figure 12 (a) illustrate that $\theta_{0}$ affects not only the thickness of the circular arc area but also the thickness of flange, and the thinning is highly sensitive to parameter $\theta_{0}$.
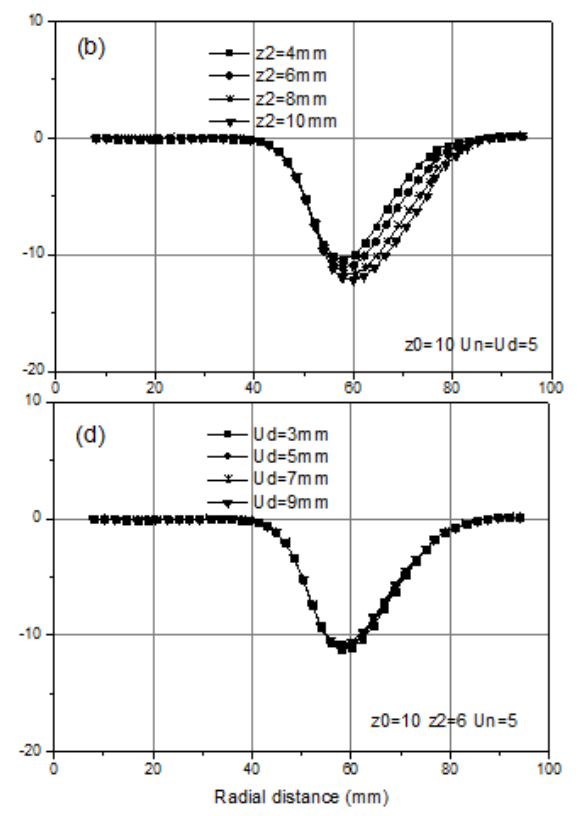

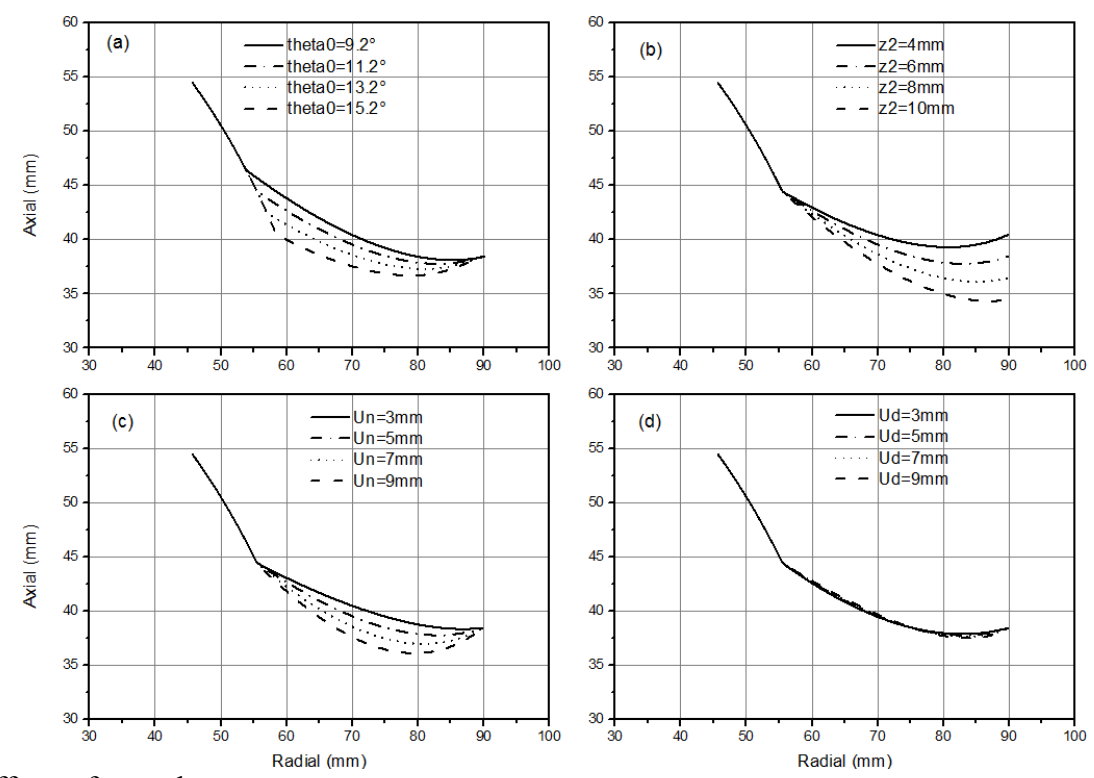

Figure 13. Shape of different forward passes

The sloping degree of the flange is defined by $Z_{2}$, while the increasing of $U_{n}$ leads to a more concave toolpath. All the starting points of the Bezier curves shown in Figure 13 (b) and (c) have the same radial coordinate of 55.4. As shown in Figure 12 (b), the increasing of $Z_{2}$ only affects the thickness of the flange, and $U_{n}$ has the similar effects on thinning. However, the wall thickness reduction is insensitive to $U_{d}$. As revealed in Figure $13(\mathrm{~d})$, with the change of $U_{d}$, the shape of toolpaths have very little variations. We can conclude that the shape of tool path, which results in thinning, is insensitive to parameter $U_{d}$.

Considering the wall thickness variation, this study tries to find a more satisfying path, which has a wellproportioned distribution of thickness, to conduct the following analysis. The expected path is chosen from the sample points selected by the design of experiments method. This paper presents the Latin hypercube as a sampling strategy. Latin hypercube sampling (LHS) [13] is a stratified random procedure that provides an efficient
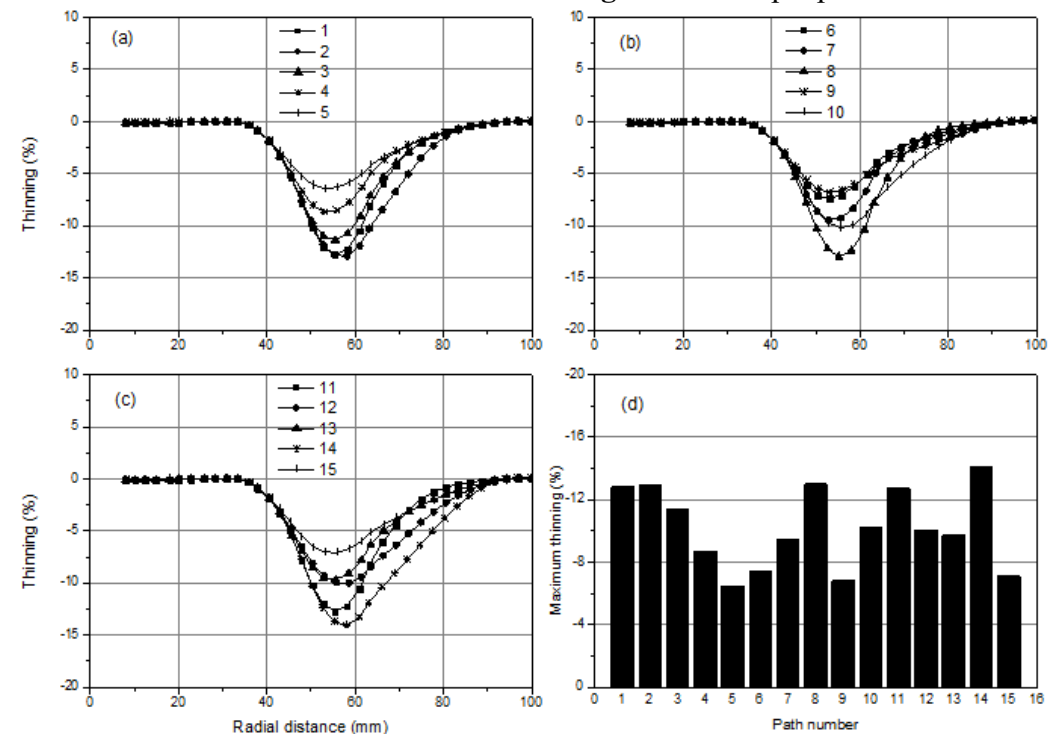

Figure 15. Thickness variation of sample points

way of sampling variables from their multivariate distributions. It provides a full coverage of the range of each variable by maximally stratifying the marginal distribution. In this section, the abovementioned 4 parameters are chosen as variables, the range of each variable is equally divided into 15 intervals, then 15 sample points are selected. The sample points' distribution is shown in Figure 14, and the $X$ and $Y$ axes represent $Z_{2}$ and $U_{d}$ respectively.

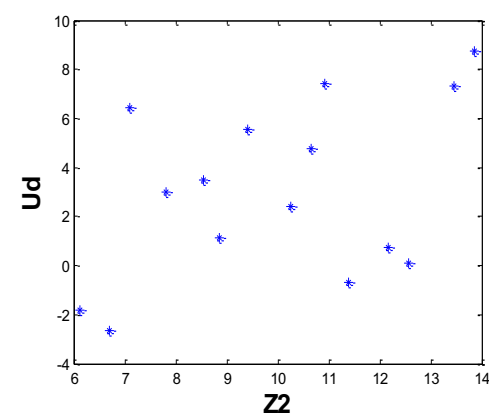

Figure 14. Sample points' distribution

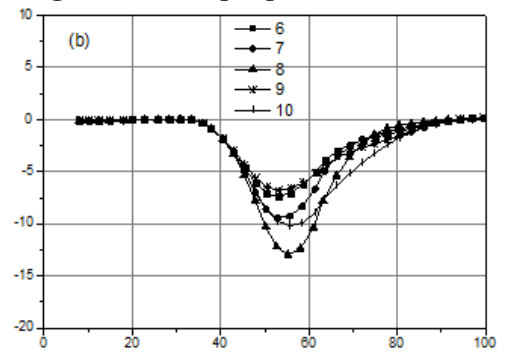

Path number 

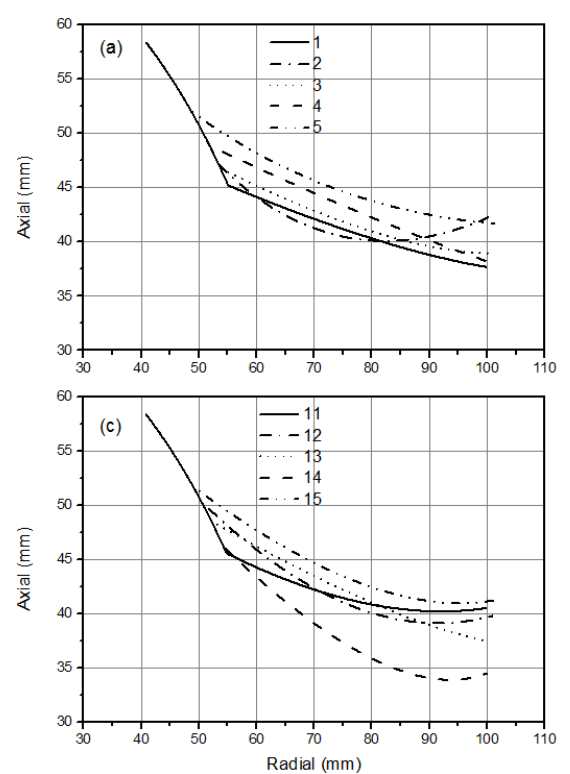

Figure 16. Roller paths of different sample points

As can be seen in Figure 15 (d), in terms of the maximum wall thickness reduction path 5 has the best result, but path 14 has the worst result. Analyzing Figure 15 we summarize that path 5,9 and 15 get the thinning of below $8 \%$, while path $1,2,8,11$ and 14 get the thinning of above $12 \%$. Comparing different shape of roller paths in Figure 16 and Table 3, we find that path 5, 9 and 15 all have small values of $\theta_{0}$, in addition, path $1,8,11$ and 14 have large values of $\theta_{0}$. For path 2, a large value of $U_{n}$ induces the serious thinning of wall thickness, although the value of $\theta_{0}$ is relatively small. Path 5 gets the best result due to the smallest value of $\theta_{0}$ and a small value of $U_{n}$. Moreover, a large value of $\theta_{0}$ and a very large value of $U_{n}$ result in the worst wall thickness reduction of path 14. The above results of tool paths indicate that the deformation of adjacent paths $\left(\theta_{0}\right)$ has a crucial influence on thinning, and another important factor is $U_{n}$, the increasing of which will lead to a more aggressive toolpath.

Table 3. Sample points of LHS (unit: $\mathrm{mm}$ )

\begin{tabular}{ccccc}
\hline & $\theta_{0}$ & $Z_{2}$ & $U_{n}$ & $U_{d}$ \\
\hline 1 & $15.61^{\circ}$ & 10.66 & 1.39 & 4.77 \\
2 & $13.56^{\circ}$ & 6.12 & 8.72 & -1.83 \\
3 & $13.93^{\circ}$ & 9.41 & 2.71 & 5.53 \\
4 & $11.66^{\circ}$ & 10.26 & 0.79 & 2.41 \\
5 & $8.08^{\circ}$ & 6.69 & 4.33 & -2.64 \\
6 & $10.67^{\circ}$ & 13.46 & -2.67 & 7.31 \\
7 & $12.96^{\circ}$ & 12.57 & -1.98 & 0.09 \\
8 & $16.25^{\circ}$ & 11.40 & -0.88 & -0.68 \\
9 & $9.30^{\circ}$ & 12.17 & 0.19 & 0.75 \\
10 & $11.25^{\circ}$ & 8.86 & 5.65 & 1.15 \\
11 & $15.17^{\circ}$ & 7.82 & 3.79 & 2.99 \\
12 & $9.98^{\circ}$ & 8.55 & 8.18 & 3.51 \\
13 & $12.00^{\circ}$ & 10.92 & 2.16 & 7.42 \\
14 & $14.61^{\circ}$ & 13.85 & 7.18 & 8.74 \\
15 & $8.36^{\circ}$ & 7.11 & 6.04 & 6.42 \\
\hline
\end{tabular}

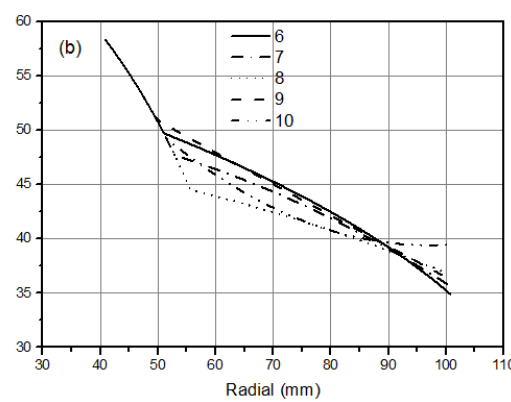

\section{Conclusions}

Based on the Finite Element model with parameterized 2pass conventional spinning process, the effects of tool path with quadratic Bezier curve on the thickness variation have been studied thoroughly. The following conclusions can be drawn from the process analysed above:

1. When the original blank is a plate, the first path in conventional spinning has significant effect on wall thickness distribution, and the toolpath with a clearance that is less than the original thickness of the blank, can obtain a good thickness result to avoid failures such as wrinkling or fracture.

2. For forward pass, the larger value of the parameters of $\theta_{0}, Z_{2}$ and $U_{n}$, the more severe the thinning phenomenon is. It is interpreted like this: the increase of the deformation between adjacent passes, the increase of the sloping degree of the flange and the more aggressive of the Bezier curve all result in a more serious thinning.

These conclusions could be used to conduct processes with multi-pass conventional spinning to produce better dimensional precision of spherical components.

\section{Acknowledgement}

The research was supported by National Basic Research Program of China (973 Program, No.2014CB046601).

\section{References}

1. C.C. Wong, T.A. Dean, J. Lin. International Journal of Machine Tools \& Manufacture, 43 (2004): 14191435 .

2. M. Runge. Spinning and flow forming. Werkzeugmaschinenbau/Verlag Moderne Industrie AG, D-86895 (1994). 
3. K. Lange, Handbook of metal forming, McGraw-Hill Book Company, 1216 (1985).

4. M. Hayama, Hi. Kudo, T. Shinokura. Bulletin of JSME, 13(1970) : 1358-1366.

5. M. Hayama, M. Nakamura, T. Watanabe, H. Hamano. JSTP, 27(1986): 1053-1059.

6. M. Hayama. JSTP, 30:1403-1410

7. D.C. Kang, X.C. Gao, X.F. Meng, et al. Journal of Materials Processing Technology. 91(1999): 226230.

8. L. Wang, H. Long. Journal of Materials Processing Technology, 211(2011) : 2140-2151.

9. Y. Li, J. Wang, G. Lu, et al. Journal of Zhejiang University SCIENCE A, 15(2014): 432-446.

10. J.A. Polyblank, J.M. Allwood. CIRP AnnalsManufacturing Technology, 64(2015) : 301-304.

11. E. Quigleg, J. Monaghan. Journal of Materials Processing Technology, 103(2000) : 114-119.

12. Q.X. Xia, X.Q. Cheng, Y. Hu, F. Ruan. International Journal of Mechanical Sciences, 48(2006) : 726-735.

13. M.D. McKay, R.J. Beckman, W.J. Conover. Technometrics, 21(1979) : 239-245. 